\title{
Autoestima, Apoyo Social y Satisfacción Vital en Adolescentes
}

\author{
Self Esteem, Social Support and Life Satisfaction in Adolescents
}

\author{
Juan Luis San Martín \\ Universidad Santo Tomás, Chile \\ Enrique Barra \\ Universidad de Concepción, Chile
}

(Rec: 15 de agosto de 2012 / Acept: 19 de Agosto de 2013)

\begin{abstract}
Resumen
Este estudio examinó la relación de las variables autoestima y apoyo social con la satisfacción vital en una muestra de 512 adolescentes de ambos sexos, con edades entre 15 y 19 años (promedio de 16.4 años), de la ciudad de Concepción (Chile). Los resultados mostraron que tanto la autoestima como el apoyo social tenían relaciones positivas significativas con la satisfacción vital. El análisis de regresión reveló que ambas variables predecían significativamente el nivel de satisfacción vital, siendo mayor la influencia relativa del apoyo social que de la autoestima. Se proponen diversas explicaciones para los resultados obtenidos.

Palabras clave: satisfacción vital, autoestima, apoyo social, adolescentes
\end{abstract}

\begin{abstract}
This study examined the relationship between self-esteem, perceived social support and life satisfaction in a sample of 512 male and female adolescents from the city of Concepcion (Chile) between 15 and 19 years. Both self-esteem and social support had significant positive relationships with life satisfaction. Regression analysis showed that both factors predicted life satisfaction, with perceived social support having more influence on life satisfaction than self-esteem. Diverse explanatory factors for obtained results are proposed. Key words: life satisfaction, self esteem, social support, adolescents
\end{abstract}

\section{Introducción}

En consonancia con el gran desarrollo que ha alcanzado la Psicología Positiva, durante los últimos años ha aumentado el interés de los investigadores por los aspectos positivos de la adaptación psicológica de los adolescentes, lo cual ha llevado a investigar en esta etapa del desarrollo temas como la calidad de vida (Avendaño y Barra, 2008; Urzúa y Mercado, 2008) y el bienestar subjetivo (Castellá et al., 2012; Dinisman, Monserrat y Casas, 2012; Tomyn y Cummins, 2011; Verdugo et al., 2013). Dentro de los componentes del concepto de bienestar subjetivo, la satisfacción vital es el elemento más cognitivo y corresponde a la evaluación global respecto a la totalidad de la propia vida (Antaramian y Huebner, 2009; Diener, Scollon y Lucas, 2009).
De acuerdo a Huebner y Diener (2008), aquellos adolescentes que informan altos niveles de satisfacción vital tienden a mostrar además mejores niveles de funcionamiento en las áreas intrapersonal, interpersonal y en el área escolar. Por el contrario quienes se encuentran extremadamente insatisfechos con sus vidas, presentan dificultades importantes que incluyen conducta agresiva, ideación suicida, conductas sexuales de riesgo, consumo de alcohol o drogas, problemas físicos y de alimentación, e inactividad física. La mayor parte de los adolescentes refieren estar satisfechos con sus vidas y con determinados dominios específicos como familia, amistades y escuela (Huebner y Diener, 2008), resultados que en términos generales se replican en distintas culturas (Gilman et al., 2008). 
Respecto a los predictores de la satisfacción vital, la investigación indica que las variables demográficas como edad y sexo aportan en forma modesta a la variabilidad en la satisfacción vital global de niños y adolescentes y en algunos casos no producen efectos significativos (Huebner y Diener, 2008; Proctor, Linley y Maltby, 2009). En cambio los factores disposicionales parecen ser mejores predictores de la satisfacción vital en adolescentes, existiendo evidencia que las variables ligadas al sentido de valor personal, control ambiental y competencia social se asocian a mayores niveles de satisfacción (Proctor et al., 2009).

Una de las variables disposicionales que se han estudiado en relación con la satisfacción vital es la autoestima, la cual implica una actitud hacia sí mismo que se relaciona con la creencias respecto a las propias habilidades, relaciones sociales y futuro (Rey, Extremera y Pena, 2011). Estudios revisados por Cummins y Nistico (2002) reportan relaciones significativas entre satisfacción y autoestima en distintas poblaciones, incluyendo a los adolescentes. Además estos autores han planteado que la alta autoestima constituiría uno de los sesgos positivos que contribuirían a la estabilidad en los reportes de satisfacción a lo largo del tiempo.

La relación entre autoestima y satisfacción vital no sería homogénea en distintas sociedades sino que estaría mediada por variables de tipo cultural, encontrándose que en culturas individualistas esta relación es más alta que en culturas colectivistas, mientras que en estas últimas cobra mayor importancia la calidad de las relaciones con los otros (Kang, Shaver, Sue, Min y Jing, 2003).

Otra variable asociada al bienestar en adolescentes es el apoyo social (Chu, Saucier y Hafner, 2010). Este concepto corresponde a la percepción que tiene una persona de que cuidan de ella, que es estimada y que forma parte de una red de personas que se preocupan de su bienestar (Barra, 2004). En el caso de niños y adolescentes algunas fuentes de apoyo que aportan al bienestar son la familia, los amigos y el personal escolar (Chu et al., 2010; Proctor et al., 2009; Valois, Zullig, Huebner y Drane, 2009).

El apoyo social puede ser de distintos tipos incluyendo el apoyo emocional, de consejo e instrumental (Méndez y Barra, 2008). El apoyo emocional se refiere a las conductas de cuidado de los demás, la posibilidad de compartir pensamientos y experiencias personales. El consejo consiste en la entrega de información o guías para afrontar las distintas demandas del entorno. El apoyo instrumental corresponde a la entrega de ayuda material o servicios a alguien que lo requiere.

El apoyo social se relacionaría con el bienestar debido a que promueve emociones positivas, un sentido de valor personal, contribuye a que la propia vida sea más predecible, y además actúa como un amortiguador del estrés al fortalecer la autoestima, autoeficacia y conductas relacionadas con la resolución de problemas (Barra, 2004; Tomyn y Cummins, 2011). Además es considerado un recurso externo que, al estar presente junto a otras variables en los adolescentes, disminuye la participación en conductas que dañan la salud y promueve la posibilidad de alcanzar éxito en distintos contextos (Valois et al., 2009).

Aunque no se conocen estudios que exploren específicamente la relación entre el apoyo social y la satisfacción vital en adolescentes chilenos, existen antecedentes que aportarían algunas evidencias indirectas sobre esta relación. Por una parte Barra, Cancino, Lagos, Leal y San Martín (2005), utilizando la misma medida de apoyo social del presente estudio, encontraron que el apoyo social percibido mostraba una baja asociación inversa con el ánimo depresivo y con el reporte de problemas de salud en adolescentes. Por otra parte, Barra, Cerna, Kramm y Véliz (2006), utilizando una medida diferente de apoyo social, encontraron que en los adolescentes estudiados no había relación significativa entre el apoyo social y las variables problemas de salud, ánimo depresivo y estrés percibido. Los autores de este estudio mencionan como una de las posibles causas de este resultado los cambios en las estructuras de apoyo que tienen lugar en la adolescencia, lo que produciría inestabilidad en las percepciones de apoyo. Por último, Avendaño y Barra (2008) informaron que en una muestra de adolescentes con enfermedades crónicas, el apoyo social percibido mostró tener poca influencia en la calidad de vida reportada, en comparación con la variable autoeficacia.

Dada la relativa escasez de estudios que exploren el funcionamiento psicológico positivo en adolescentes (Huebner y Diener, 2008) y que en esta etapa las variables psicológicas individuales se encuentran todavía en desarrollo (Klimstra, Hale, Raaijmakers, Branje y Meeus, 2009; Zeiders, Umaña-Taylor y Derlan, 2013), surge la necesidad de estudiar de manera específica la satisfacción vital en adolescentes. Además, debido a que se ha informado que los factores culturales moderan la relación entre las variables predictoras y la satisfacción vital (Gilman et al., 2008), resulta importante estudiar el grado en que los hallazgos sobre satisfacción vital en adolescentes de otros países se replican en nuestra realidad.

La presente investigación se propuso como objetivos: a) examinar las relaciones entre las variables satisfacción vital, autoestima y apoyo social, y b) identificar el aporte relativo de la autoestima y el apoyo social en la satisfacción vital de los adolescentes. 


\section{Método}

\section{Participantes}

La muestra estuvo compuesta por 512 participantes (57.2\% hombres y $42.8 \%$ mujeres), alumnos de $2^{\circ}$ y $3^{\circ}$ Medio de 7 establecimientos educacionales de la ciudad de Concepción (Chile). De ellos, un 43.4 por ciento asistía a dos establecimientos municipales, un 43.1 por ciento a cuatro establecimientos particulares subvencionados y un 13.5 por ciento a un colegio privado. La edad de los participantes varió entre 15 y 19 años con un promedio de 16.4 años.

\section{Instrumentos}

Escala Multidimensional de Satisfacción Vital para Estudiantes (Huebner, Laughlin, Ash y Gilman, 1998): consta de 40 ítems que miden distintas dimensiones de la satisfacción vital, incluyendo familia, amigos, escuela, entorno y sí mismo. Cada ítem cuenta con 5 opciones de respuesta que van desde "muy en desacuerdo" a "muy de acuerdo". Para este estudio la escala original se tradujo y adaptó con la asesoría de un hablante nativo del idioma inglés experto en construcción de instrumentos. En la muestra estudiada se obtuvo un valor de confiabilidad medida a través del alfa de Cronbach de 0.89 para el instrumento total, de 0.90 para la satisfacción familiar, de 0.82 para la satisfacción con los amigos, de 0.81 para la satisfacción escolar, de 0.79 para la satisfacción con el entorno y de 0.85 para la satisfacción con el sí mismo.

Escala de Autoestima de Rosemberg, versión española (Martín, Núñez, Navarro y Grijalva, 2007): escala de autoreporte tipo Likert de 10 ítems, cuyos contenidos se centran en los sentimientos de respeto y aceptación de sí mismo. Cada ítem consta de cinco posibles respuestas. La puntuación total varía entre 10 y 50 puntos donde los puntajes mayores indican mayor autoestima. El coeficiente alfa de Cronbach obtenido en el presente estudio fue de 0.79 .

Escala Multidimensional de Apoyo Social Percibido (CantyMitchell y Zimet, 2000): compuesta por 12 ítems que recogen información del apoyo social percibido por los individuos en tres áreas: familia, amigos y otros significativos. Posee 5 alternativas de respuesta, que varían entre muy en desacuerdo y muy de acuerdo. En el presente estudio se obtuvo una consistencia interna mediante coeficiente alfa de Cronbach de 0.90.

\section{Procedimiento}

Los instrumentos fueron primero aplicados a un grupo piloto de 27 adolescentes de características similares a los de la muestra final. Este procedimiento permitió identificar algunas dificultades y establecer la confiabilidad de los instrumentos, la cual resultó adecuada. Se solicitó la autorización de los directores en los establecimientos y se obtuvo el consentimiento informado de los adolescentes. El estudio se planteó como una actividad voluntaria, los adolescentes que no deseaban participar podían retirarse. La aplicación de los instrumentos se realizó de forma colectiva, durante horas de clases.

\section{Resultados}

Con el objeto abordar el primer objetivo de este estudio, se calcularon las correlaciones lineales entre satisfacción vital, autoestima y apoyo social percibido, tanto en la muestra total como en cada sexo, mediante el coeficiente de Pearson.

Tabla 1. Correlaciones entre las variables en la muestra total

\begin{tabular}{lccc}
\hline & 1 & 2 & 4 \\
\hline 1. Satisfacción vital & - & $0.53^{* *}$ & $0.64^{* *}$ \\
2. Autoestima & & - & $0.34^{* *}$ \\
3. Apoyo social percibido & & & - \\
\hline
\end{tabular}

Nota. $* * \mathrm{p}<.01$

Como se aprecia en la Tabla 1, a nivel de la muestra total tanto la autoestima como el apoyo social presentan relaciones significativas con la satisfacción vital, siendo el apoyo social el que muestra la mayor relación.

Tabla 2. Correlaciones entre las variables en cada sexo

\begin{tabular}{lccc}
\hline & 1 & 2 & 4 \\
\hline 1. Satisfacción vital & - & $0.54^{* *}$ & $0.63^{* *}$ \\
2. Autoestima & $0.54^{* *}$ & - & $0.33^{* *}$ \\
3. Apoyo social percibido & $0.66^{* *}$ & $0.35^{* *}$ & -
\end{tabular}

Se presentan los coeficientes de correlación para los hombres en la parte superior de la diagonal y para las mujeres en la parte inferior. Nota. $* * p<.01$

En la Tabla 2 se puede observar que, el patrón de correlaciones en cada sexo es en general similar al encontrado en la muestra total, no apreciándose diferencias importantes entre ambos sexos

Con el propósito de cumplir con el segundo objetivo se realizó un análisis de regresión lineal estándar con la 
Tabla 3. Coeficientes de regresión

\begin{tabular}{|c|c|c|c|c|c|c|}
\hline & \multirow[b]{2}{*}{$\beta$} & \multirow{2}{*}{\multicolumn{2}{|c|}{$t$ Sig. }} & \multicolumn{3}{|c|}{ Correlaciones } \\
\hline & & & & $\begin{array}{c}\text { Orden } \\
\text { cero }\end{array}$ & Parcial & $\begin{array}{l}\text { Semi- } \\
\text { parcial }\end{array}$ \\
\hline $\begin{array}{l}\text { Apoyo } \\
\text { social }\end{array}$ & 0.52 & 15.57 & .000 & 0.64 & 0.58 & 0.49 \\
\hline Autoestima & 0.34 & 10.29 & .000 & 0.52 & 0.42 & 0.32 \\
\hline
\end{tabular}

Nota. Variable dependiente: Satisfacción vital, R: $0.72 \mathrm{R}^{2}$ ajustado: 0.52

satisfacción vital como variable criterio y como variables predictoras la autoestima y el apoyo social. La Tabla 3 presenta los coeficientes de regresión estandarizados (beta) con sus valores $t$ asociados, los coeficiente de correlación de orden cero, parcial y semiparcial.

Se observa en la Tabla 3 que los coeficientes de regresión tanto del apoyo social como de la autoestima resultan altamente significativos, siendo el apoyo social el predictor más importante de la satisfacción vital de los adolescentes. En conjunto ambas variables predictoras explicarían un 52\% de la variabilidad en la satisfacción vital.

\section{Discusión}

El primer objetivo de esta investigación fue examinar las relaciones entre las variables estudiadas. El análisis de correlación reveló que la satisfacción vital de los adolescentes mostró asociaciones positivas significativas tanto con la autoestima como con el apoyo social percibido, hallazgos concordantes con lo reportado en la literatura (Kapıkıran, 2013; Kong y You, 2013: Proctor et al., 2009). La magnitud de la relación observada entre autoestima y satisfacción vital se encuentra en el rango de lo encontrado en estudios previos (Rey et al., 2011), mientras que el grado de relación encontrada entre el apoyo social y la satisfacción vital es mayor al que podría haberse esperado a partir de la literatura (Cummins y Nistico, 2002), lo que constituye uno de los hallazgos más interesantes de este estudio. Este resultado difiere del reporte de otras investigaciones que incluían la autoestima y otras variables similares al apoyo social percibido (calidad de las relaciones, satisfacción con las relaciones), las cuales encontraron que era la autoestima la que presentaba las asociaciones de mayor magnitud con la satisfacción vital y que esta relación era más acentuada en culturas individualistas (Kang et al., 2003).
Cabe señalar además que no se detectaron diferencias importantes entre ambos sexos en las correlaciones entre las variables estudiadas, lo que indicaría que tanto en hombres como en mujeres las variables relacionadas con la satisfacción vital son similares.

En cuanto al segundo objetivo, consistente en examinar la influencia relativa de la autoestima y el apoyo social sobre la satisfacción vital, los resultados indican que ambas variables predecirían una proporción importante de la variabilidad en la satisfacción vital, siendo de mayor magnitud la influencia del apoyo social.

Respecto de esta mayor influencia del apoyo social percibido por sobre la autoestima se proponen algunas explicaciones. En primer lugar se pueden mencionar las características de los instrumentos utilizados en este estudio para medir satisfacción vital y apoyo social percibido. En ambos casos se trata de medidas multidimensionales que examinan entre otras las áreas familia y amistades. Es posible que el apoyo social percibido medido de esta forma resulte un mejor predictor de la satisfacción vital puesto que se indaga respecto de la percepción de apoyo en áreas que también son consideradas explícitamente en el instrumento utilizado para medir satisfacción vital.

Otra posible explicación dice relación con variables de tipo cultural. Se podría pensar que las sociedades latinoamericanas se caracterizan por una mayor orientación colectivista si se comparan con Estados Unidos y países de Europa. Es posible que esta característica contribuya a que exista una asociación más alta entre apoyo social percibido y satisfacción vital, de la que se ha reportado en estudios de otros países. Además el hecho de que durante la adolescencia se encuentren en desarrollo tanto las estructuras de apoyo (Barra et al., 2006) como la autoestima (Klimstra et al., 2009) podría explicar los resultados discordantes con la literatura respecto de la importancia relativa de esta variable como predictor de la satisfacción vital.

Pese a no ser el predictor más importante, la autoestima constituye un aporte significativo para explicar la variabilidad en la satisfacción vital de los adolescentes estudiados. Este hallazgo coincide con los estudios que han encontrado que la autoestima se relaciona con distintos indicadores de funcionamiento psicológico sano en adolescentes (Huebner y Diener, 2008; Proctor et al., 2009). La autoestima constituiría un amortiguador interno de tipo cognitivo que opera ante distintas circunstancias que pudieran amenazar la satisfacción vital de los adolescentes (Cummins, 2010), mitigando el impacto de las experiencias negativas. Los adolescentes que cuentan con mayores niveles de autoestima estarían menos expuestos a la 
influencia de esos factores externos y mantendrían así niveles de satisfacción vital relativamente altos. Por el contrario, quienes presentan un bajo nivel de autoestima pueden ver afectados negativamente sus niveles de satisfacción vital ante eventos externos ya que estos impactarían en forma más intensa.

Este tipo de mecanismos cumplen un rol adaptativo ya que para mantenerse motivados y alcanzar adecuadas condiciones de vida, los seres humanos necesitan mantener un nivel relativamente alto de satisfacción que además requiere ser estable y predecible (Cummins y Nistico, 2002). Además la baja autoestima se relacionaría con estados afectivos negativos que, si son frecuentes o están accesibles fácilmente en la memoria, podrían incidir negativamente en los juicios que los adolescentes hagan de sus vidas (Diener et al., 2009).

Como una limitación de la presente investigación cabe señalar su carácter transversal. En este estudio se propone que las variables apoyo social y autoestima influyen de manera significativa en los niveles de satisfacción, sin embargo, a partir del diseño de tipo retrospectivo utilizado no es posible descartar una influencia reciproca, por lo que el planteamiento anterior se sustenta principalmente en antecedentes teóricos. Por lo cual como sugerencia para futuras investigaciones se puede plantear la necesidad de desarrollar estudios de tipo prospectivo. Además sería deseable ampliar el rango de edades de manera de analizar la trayectoria que siguen las variables analizadas y su relación durante la etapa adolescente.

Los resultados obtenidos en el presente estudio contribuirían al conocimiento sobre los factores que se relacionan con el bienestar subjetivo de los adolescentes, aportando a la comprensión de los procesos a partir de los cuales se estructuran los juicios evaluativos sobre la propia vida que conforman la satisfacción vital. Dicha información resultaría relevante para guiar los esfuerzos por implementar medidas que contribuyan a promover niveles adecuados de bienestar de los adolescentes.

\section{Referencias}

Antaramian, S., y Huebner, S. (2009). Stability of adolescents' multidimensional life satisfaction reports. Journal of Psychoeducational Assessment, 27, 421-425.

Avendaño, M. J., y Barra, E. (2008). Autoeficacia, apoyo social y calidad de vida en adolescentes con enfermedades crónicas. Terapia Psicológica, $26,165-172$

Barra, E. (2004). Apoyo social, estrés y salud. Psicología y Salud, 14, 237-243.

Barra, E., Cancino, V., Lagos, G., Leal, P., y San Martín, J. (2005). Factores psicosociales y problemas de salud reportados por adolescentes. Psicología y Salud, 15, 231-239.

Barra, E., Cerna R., Kramm D., y Veliz, V. (2006). Problemas de salud, estrés, afrontamiento, depresión y apoyo social en adolescentes. Terapia Psicológica, 24, 55-61.
Canty-Mitchell, J., y Zimet, G. (2000). Psychometric properties of the multidimensional scale of social support in urban adolescents. American Journal of Community Psychology, 28, 391-400.

Castellá, J., Saforcada, E., Tonon, G., Rodríguez, L., Mozobancyk, S., y Bedin, L. (2012). Bienestar subjetivo de los adolescentes: un estudio comparativo entre Argentina y Brasil. Psychosocial Intervention, 21, 273-280.

Chu, P. S, Saucier, D. A., y Hafner, E. (2010). Meta-analysis of the relationships between social support and well-being in children and adolescents. Journal of Social and Clinical Psychology, 29, 624-645.

Cummins, R. A., y Nistico, H. (2002). Maintaining life satisfaction: The role of positive bias. Journal of Happiness Studies, 3, 37-69.

Diener, E., Scollon, C. N., y Lucas, R. E. (2009). The evolving concept of subjective well-being. En E. Diener (Ed.), Assesing well-being. The collected works of Ed Diener (pp. 67-100). Nueva York: Springer.

Dinisman, T., Monserrat, C., y Casas, F. (2012). The subjective wellbeing of Spanish adolescents: Variations according to different living arrangements. Children and Youth Services Review, 34, 2374-2380.

Gilman, R., Huebner, E., Tian, L., Park, N., O’Byrne, M., Schiff, M., ... Langknecht, H. (2008). Cross-national adolescent multidimensional life satisfaction reports: Analyses of mean scores and response style differences. Journal of Youth and Adolescence, 2, 142-154.

Huebner, E. S., y Diener, C. (2008). Research on life satisfaction of children and youth: Implications for the delivery of school-related services. En M. Eid y R. Larsen (Eds.), The science of subjective well-being (pp. 376-392). Nueva York: Guilford Press.

Huebner, E., Laughlin, J., Ash C., y Gilman, R. (1998). Further validation of the Multidimensional Students' Life Satisfaction Scale. Journal of Psychological Assessment, 16, 118-134.

Kang, S., Shaver, P. R., Sue, S., Min, K., y Jing, H. (2003). Culture-specific patterns in the prediction of life satisfaction: Roles of emotion, relationship quality, and self-esteem. Personality and Social Psychology Bulletin, 29, $1596-1608$.

Kapıkıran, S. (2013). Loneliness and life satisfaction in Turkish early adolescents: The mediating role of self- esteem and social support. Social Indicators Research, 111, 617-632.

Klimstra, T. A., Hale, W. W., Raaijmakers, Q. A. W., Branje, S. J. T., y Meeus, W. (2009). Maturation of personality in adolescence. Journal of Personality and Social Psychology, 96, 898-912.

Kong, F., y You, X. (2013). Loneliness and self-esteem as mediators between social support and life satisfaction in late adolescence. Social Indicators Research, 110, 271-279.

Martín, J., Núñez, J., Navarro, J., y Grijalva, F. (2007). The Rosenberg Self-Esteem Scale: Translation and validation in University students. The Spanish Journal of Psychology, 10, 458-467.

Méndez, P., y Barra, E. (2008). Apoyo social percibido en adolescentes infractores de ley y no infractores. Psykhe, 17, 59-64.

Proctor, C. L., Linley, P. A., y Maltby, J. (2009). Youth life satisfaction: A review of the literature. Journal of Happiness Studies, 10, 583-630.

Rey, L., Extremera, N., y Pena, M. (2011). Perceived emotional intelligence, self-esteem and life satisfaction in adolescents. Psychosocial Intervention, 20, 227-234.

Tomyn, A. J., y Cummins, R. A. (2011). The subjective wellbeing of high-school students: Validating the Personal Wellbeing Index-School Children. Social Indicators Research 101, 405-418.

Urzúa, A., y Mercado, G. (2008). La evaluación de la calidad de vida de los y las adolescentes a través del Kiddo-Kindl. Terapia Psicológica, 26, 133-141.

Valois, R. F., Zullig, K. J., Huebner, E. S., y Drane, J. W. (2009) Youth developmental assets and perceived life satisfaction: Is there a relationship? Applied Research Quality of Life, 4, 315-331.

Verdugo, J., Ponce de León, B., Guardado, R., Meda, R., Uribe, J., y Guzmán, J. (2013). Estilos de afrontamiento al estrés y bienestar subjetivo en adolescentes y jóvenes. Revista Latinoamericana de Ciencias Sociales, Niñez y Juventud, 11, 79-91.

Zeiders, K. H., Umaña-Taylor, A. J., y Derlan, C. (2013). Trajectories of depressive symptoms and self-esteem in Latino youths: Examining the role of gender and perceived discrimination. Developmental Psychology, 49, 951-963. 
